\title{
Biomarkers of propofol metabolism in neonates: the quest beyond ontogeny
}

\author{
Anne Smits ${ }^{*}$, , Pieter Annaert ${ }^{2}$ \& Karel Allegaert ${ }^{3,4}$ \\ ${ }^{1}$ Neonatal intensive care unit, University Hospitals Leuven, Leuven, Belgium \\ ${ }^{2}$ Drug Delivery \& Disposition, Department of Pharmaceutical \& Pharmacological Sciences, KU Leuven, Leuven, Belgium \\ ${ }^{3}$ Department of Development \& Regeneration, KU Leuven, Leuven, Belgium \\ ${ }^{4}$ Intensive Care \& Department of Pediatric Surgery, Erasmus MC-Sophia Children's Hospital, Rotterdam, The Netherlands \\ * Author for correspondence: Tel.: +32 1634 3565; anne.smits@uzleuven.be
}

\section{"We anticipate that the contribution of genetic variants will be different in neonates compared with adults, since ontogeny of the metabolizing enzymes has to be taken into account"}

First draft submitted: 1 June 2017; Accepted for publication: 11 July 2017; Published online: 23 October 2017

Keywords: covariate $\bullet$ genetic variants $\bullet$ metabolism $\bullet$ neonate $\bullet$ ontogeny $\bullet$ pharmacodynamics $\bullet$ pharmacokinetics - postmenstrual age $\bullet$ postnatal age $\bullet$ propofol

The use of propofol, a short-acting sedative, for procedural sedation in neonates is increasing. In early life, a remarkable variability in propofol clearance has been documented. At present, two-third of this variability can be explained by age [1]. Although the impact of indirect hyperbilirubinemia as biomarker of reduced propofol clearance in neonates was explored, it was not retained as relevant clinical predictor [2]. Since in the last few years, no additional covariates of propofol pharmacokinetics (PK) and/or pharmacodynamics (PD) have been documented in neonates, it might be useful to determine whether covariates defined in other populations are relevant to be explored in neonates or not. Starting with a summary of covariates contributing to propofol PK/PD variability in adults, we reflect on the current knowledge of propofol disposition in neonates, and on future challenges to further explore covariates explaining neonatal propofol disposition.

As a highly lipophilic compound, propofol immediately distributes to the subcutaneous fat and central nervous system after intravenous administration. In adults, $90 \%$ of the propofol dose is metabolized in the liver, and the metabolites are excreted in urine. Both Phase I (hydroxylation) and Phase II (glucuronidation) processes contribute to propofol metabolism. In adults, $70 \%$ is converted to propofol glucuronide by uridine diphosphate UGT1A9, while the CYP2B6 and to a lesser extent, CYP2C9 are responsible for the hydroxylation of propofol to 1- and 4quinol metabolites. These metabolites subsequently undergo glucuronidation or sulfation [3]. The large intersubject variability in propofol biotransformation in adults is in part attributed to patient-related characteristics (i.e., age, weight, height, lean body mass, gender and genetic polymorphisms), disease state and environmental factors.

Choong et al. documented the impact of gender in adults receiving propofol by target-controlled infusion for elective surgery. Significantly higher area under the curve values of the hydroxyl and glucuronide metabolites were observed in women [4]. This suggests a dimorphic propofol metabolism in vivo, with higher metabolic turnover in female compared with male subjects. Their findings confirmed results of a previous pilot study as well as in vitro data [5]. In their pilot study, higher (i.e., 1.7 to 2.1-fold difference) weight-corrected area under the curve for all propofol glucuronides after a single propofol bolus were found in women compared with men [5]. Also in an elderly cohort (65-91 years), the impact of gender on propofol PK was observed by Vuyk et al. Predicted propofol concentrations during continuous infusion (i.e., $1.5 \mathrm{mg} / \mathrm{kg}$ propofol bolus in $1 \mathrm{~min}$ followed by $7 \mathrm{mg} / \mathrm{kg} / \mathrm{h}$ for $89 \mathrm{~min}$ ) were $10-15 \%$ higher in men compared with women, highlighting that elderly female patients should be given 10\% higher infusion rates compared with males to achieve the same propofol blood concentrations [6]. Safe propofol use also warrants the recognition of the impact of disease/injury-related variables on its disposition. Population PK analysis of a propofol bolus in adult burn patients (with mean \pm SD: $41 \pm 19 \%$ total body surface area burns) compared with nonburn controls observed increased clearance (population mean estimates for the clearances in the central [ $4.19 \mathrm{vs} 1.74 \mathrm{l} / \mathrm{min}$ ] and slow peripheral compartments [3.56 vs $1.09 \mathrm{l} / \mathrm{min}$ ] for burn 
vs nonburn, respectively) and expanded volume of distribution in the central compartment (population mean estimate: 48.4 vs 27.6 1) in the burn cohort. Burn was the most important covariate, followed by covariate weight. These results were attributed to altered global and microvascular organ perfusion and redistribution of blood flow in the hyperdynamic phase of the burn injury recovery. Also, large volumes of fluid resuscitation, increased tissue edema and third space effects might contribute to the increased distribution volume [7].

While reports on the impact of clinical characteristics and gender on the variation in metabolic clearance of propofol are quite consistent, the contribution of genetic polymorphisms requires further exploration. Besides the genes encoding for CYP2B6, CYP2C9 and UGT1A9 enzymes, the GABRG2, GSTP1, SULT1A and NQO1 genes are also considered of relevance for propofol PK and/or PD [3]. In 101 adult Caucasian patients (mean age: $32.5 \pm 9.6$ years) receiving propofol infusion for elective surgery, the impact of single-nucleotide polymorphisms in CYP2B6, CYP2C9, UGT1A9 as well as in the GABRE gene (encoding the GABA-A receptor) on propofol dose, depth of anesthesia (measured by electroencephalography) and clinical recovery was investigated [3]. According to the authors, none of the most common genetic variants analyzed in that study could explain the large variability in propofol PK and response to the drug. However, they did find that patients with UGT1A9-331C/T had a higher propofol clearance and needed a higher propofol induction dose [3]. Patients with UGT1A9-1818T/C required a longer time to loss of consciousness and those with $C Y P 2 C 9^{*} 2 /{ }^{*} 2$ had a higher propofol plasma concentration than the other patients. In addition, Kansaku et al., described CYP2B 6 and UGT1A9 genotype as determinant covariates of propofol PK and subsequent PD [8]. Very recently, CYP2B6 genotype-based propofol dosing was presented as a theoretical approach to improve drug exposure. Dose adjustments for elderly patients with CYP2B6 AA and AG genotype, would hereby result in a 50\% decrease in infusion dose to achieve optimal propofol effect [9]. This nicely illustrates the relevance but also the limitations of pharmacogenetics in clinical pharmacology. Taking into account the clinical effects of propofol, polymorphisms in the propofol target (GABA-A receptor) would likely have had an impact on the onset of sedation (e.g., loss of consciousness), while polymorphisms affecting the clearance of propofol would be expected to have an impact on clinical recovery. One should be aware that these 'end points' remain imprecise and definitions often vary between studies [3].

Due to specific characteristics of the neonatal population, observations on drug disposition in adults cannot just be extrapolated to neonates. Besides differences in body composition, neonates have immature hepatic and renal function compared with older children and adults. Based on population PK analyses, overall propofol clearance is lower in neonates compared with adults, displays large variability with hydroxylation as most important route, and with postmenstrual age (PMA) as the most relevant covariate. In addition, a postnatal age (PNA) of 10 days was defined as a time point after which glucuronidation capacity increases $[1,10,11]$. As mentioned before, populationspecific diseases like indirect hyperbilirubinemia could not be retained as a relevant biomarker of reduced propofol clearance in neonates, and the same holds true for gender [2]. At present, covariates PMA and PNA explain 67\% of interindividual variability in propofol clearance. To explain the remaining $33 \%$ of the observed clearance variability, the search for new covariates or biomarkers needs to continue. Pharmacogenetics, considered as a tool for precision medicine also in neonates, may play a role in this search. However, the usefulness of genetic variants as covariates is limited to periods in development where genotype-phenotype correlation is already present [12]. Furthermore, early life is characterized by isoenzyme-specific maturation of activity. There is a two to threefold increase in CYP2C9 expression in the neonatal period compared with the third trimester of fetal life, achieving $25 \%$ levels of those in adults [13]. Also for CYP2B6 and UGT1A9 ontogeny, a progressive increase in expression and/or activity in infancy has been described [14,15]. As a conclusion, given the rather moderate impact of genetic variants on propofol metabolism in adults, we anticipate that their contribution in neonates will be different because the ontogeny of the metabolizing enzymes has to be taken into account. Since hydroxylation is the main propofol metabolic pathway in neonates, a future pharmacogenetic analysis should primarily focus on CYP2B6 and CYP2C9.

The same holds true for the impact of hepatic blood flow (HBF). Liver size seems to be an indicator of glucuronidation activity for the UGT2B7 substrates, morphine and zidovudine. The impact of HBF on the clearance for different age groups obviously also depends on the substrate $(<5 \%$ for morphine; $3.7-7.9 \%$ for zidovudine) considered. However, this model failed in the first 2 weeks of postnatal life, either due to maturational changes in UGT2B7 or HBF in early neonatal life [16]. As enzyme activities in the neonatal liver are often (but not exclusively) beginning to maturate (which corresponds to low intrinsic clearance values), it becomes less likely that overall hepatic clearance in neonates will be limited by HBF. Nevertheless, neonatal HBF values have previously been scaled from adult values normalized for liver tissue mass [17] and cross-validation of these values remains very limited to date [18]. 
"We anticipate that the contribution of genetic variants will be different in neonates compared with adults, since ontogeny of the metabolizing enzymes has to be taken into account."

These all aspects illustrate that ontogeny, reflected by PMA and PNA, remains the most important determinants of neonatal propofol metabolism. Current suggestions for 'add-on' covariates (e.g., disease, genotype, liver blood flow, extrahepatic metabolism, environmental factors, enzyme inducers) have to be evaluated and integrated with the major covariate age. This search 'beyond ontogeny' will further be complicated by the rapid evolution on neonatal intensive care. New techniques and approaches toward less invasive therapy are being introduced. New approaches requiring short acting sedatives often include their own PD end points. Although the major covariates of propofol metabolism will remain the same in these new approaches and/or indications, subsequent translation to clinical practice will be more difficult. Multicenter pooling of datasets will be necessary to make progress in this 'quest beyond ontogeny'. Although it is interesting to unravel covariates of propofol PK in neonates, we want to stress that it is the concentration-effect relation in the individual patient that finally matters from a clinical perspective. Therefore, the study of circulation, perfusion, autoregulation and the development of validated measurement tools to assess these parameters, might not only provide basic physiological data (e.g., liver blood flow) which can be used in 'simple' physiology-based PK (PBPK) models, but will also support more predictive physiology-based PK and PD assessment of short-acting compounds like propofol in neonates [19].

\section{Financial \& competing interests disclosure}

The research activities of $K$ Allegaert are facilitated by the agency for innovation by Science and Technology in Flanders (IWT) through the SAFEPEDRUG project (IWT/SBO 130033). The authors have no other relevant affiliations or financial involvement with any organization or entity with a financial interest in or financial conflict with the subject matter or materials discussed in the manuscript apart from those disclosed.

No writing assistance was utilized in the production of this manuscript.

\section{References}

1 Allegaert K, Peeters MY, Verbesselt R et al. Interindividual variability in propofol pharmacokinetics in preterm and term neonates. $\mathrm{Br}$. J. Anaesth. 99, 864-870 (2007).

2 Smits A, De Cock RF, Cossey V, Knibbe CA, Allegaert K. Is indirect hyperbilirubinemia a useful biomarker of reduced propofol clearance in neonates? Biomark. Med. 6, 283-289 (2012).

3 Khan MS, Zetterlund EL, Green $\mathrm{H}$ et al. Pharmacogenetics, plasma concentrations, clinical signs and EEG during propofol treatment. Basic Clin. Pharmacol. Toxicol. 115, 565-570 (2014).

4 Choong E, Loryan I, Lindqvist M et al. Sex difference in formation of propofol metabolites: a replication study. Basic Clin. Pharmacol. Toxicol. 113, 126-131 (2013).

5 Loryan I, Lindqvist M, Johansson I et al. Influence of sex on propofol metabolism, a pilot study: implications for propofol anesthesia. Eur. J. Clin. Pharmacol. 68, 397-406 (2012).

6 Vuyk J, Ostwouder CJ, Vletter AA, Burm AG, Bovill JG. Gender differences in the pharmacokinetics of propofol in elderly patients during and after continuous infusion. Br. J. Anaesth. 86, 183-188 (2001).

7 Yamashita S, Kaneda K, Han TH. Population pharmacokinetics of a propofol bolus administered in patients with major burns. Burns 36, 1215-1221 (2010).

8 Kansaku F, Kumai T, Sasaki K et al. Individual differences in pharmacokinetics and pharmacodynamics of anesthetic agent propofol with regard to CYP2B6 and UGT1A9 genotype and patient age. Drug Metab. Pharmacokinet. 26, 532-537 (2011).

9 Eugene AR. CYP2B6 genotype guided dosing of propofol anesthesia in the elderly based on nonparametric population pharmacokinetic modeling and simulations. Int. J. Clin. Pharmacol. Toxicol. 6, 242-249 (2017).

10 Smits A, Verbesselt R, Kulo A, Naulaers G, de Hoon J, Allegaert K. Urinary metabolites after intravenous propofol bolus in neonates. Eur. J. Drug Metab. Pharmacokinet. 38, 97-103 (2013).

11 Allegaert K, Vancraeynest J, Rayyan M et al. Urinary propofol metabolites in early life after single intravenous bolus. Br. J. Anaesth. 101, 827-831 (2008).

12 Allegaert K, van den Anker JN. Neonatal pain management: still in search for the Holy Grail. Int. J. Clin. Pharmacol. Ther. 54, 514-523 (2016).

13 Hines Ronald N. Ontogeny of human hepatic cytochromes P450. J. Biochem. Mol. Toxicol. 21, 169-175 (2007).

14 Croom EL, Stevens JC, Hines RN, Wallace AD, Hodgson E. Human hepatic CYP2B6 developmental expression: the impact of age and genotype. Biochem. Pharmacol. 78, 184-190 (2009).

15 Miyagi SJ, Milne AM, Coughtrie MW, Collier AC. Neonatal development of hepatic UGT1A9: implications of pediatric pharmacokinetics. Drug Metab. Dispos. 40, 1321-1327 (2012). 


\section{Editorial Smits, Annaert \& Allegaert}

16 Krekels EHJ, Johnson TN, den Hoedt SM et al. From pediatric covariated model to semiphysiological function for maturation: Part II sensitivity to physiological and physicochemical properties. CPT Pharmacometrics Syst. Pharmacol. 1(10), e10 (2012).

17 Björkman S. Prediction of drug disposition in infants and children by means of physiologically based pharmacokinetic (PBPK) modelling: theophylline and midazolam as model drugs. Br. J. Clin. Pharmacol. 59(6), 691-704 (2005).

18 Edginton AN, Schmitt W, Voith B, Willmann S. A mechanistic approach for the scaling of clearance in children. Clin. Pharmacokinet. 45(7), 683-704 (2006).

19 Smits A, van den Anker JN, Allegaert K. Clinical pharmacology of analgosedatives in neonates: ways to improve their safe and effective use. J. Pharm. Pharmacol. 69, 350-360 (2017). 